\title{
Therapeutic Applications of Ultrasound in Neurological Diseases
}

\author{
Kang-Ho Choi ${ }^{\star, 1}$, Ja-Hae Kim ${ }^{\dagger, 1}$ \\ Department of Neurology, Chonnam National University Hospital, Chonnam National University College of Medicine*, Gwangju; Molecular \\ Imaging Center, Department of Nuclear Medicine, Chonnam National University Hospital ${ }^{\dagger}$, Gwangju, Korea
}

Ultrasonography is well recognized as an imaging tool. Recent research in the therapeutic applications of ultrasound has made great progress. Ultrasound can be widely used in ablative or nondestructive therapies, depending on the level of energy provided. In some neurological diseases, ultrasound has been used for therapeutic purposes in clinical practice and has proven effective. The use of ultrasound energy for therapy continues to expand; therapeutic ultrasound for the treatment of essential tremor has been approved by the United States. Therapeutic ultrasound has also been approved in many countries for the treatment of Parkinson's disease, neuropathic pain, and neuropsychiatric conditions, such as major depression and obsessive-compulsive disorder. This modality has the potential to treat many more neurological diseases than are currently being treated in clinical practice. Therapeutic ultrasound can be used for functional ablation of specific brain lesions, tumor ablation, neuromodulation, immunomodulation, sonothrombolysis, angiogenesis, and soft tissue healing. In addition, the effects of transient blood-brain barrier opening for the delivery of therapeutic agents and the subsequent use of sonodynamic therapy could revolutionize the treatment of neurological diseases in the future. Therapeutic applications of ultrasound have great potential to change treatment paradigms in various neurological diseases. In this review, we discuss the current clinical applications of therapeutic ultrasound, as well as the fields in which this promising technology is expected to be useful.

J Neurosonol Neuroimag 2019;11(1):62-72

Key Words: Ultrasonic; Therapy; Blood-brain barrier; Neurosurgery; Stroke
Received: April 30, 2019

Revised: May 31, 2019

Accepted: June 11, 2019

Address for correspondence: Kang-Ho Choi

Department of Neurology, Chonnam National University Hospital, 42 Jebong-ro, Donggu, Gwangju 61469, Korea Tel: $+82-62-220-6137$ Fax: +82-62-228-3461 E-mail: ckhchoikang@hanmail.net, ckhchoikang@chonnam.ac.kr

${ }^{1}$ These authors contributed equally to the manuscript.

\section{INTRODUCTION}

Ultrasound, comprising sound waves with a frequency above $20 \mathrm{kHz}$ that humans cannot hear, is used in a wide range of diagnostic imaging applications. ${ }^{1}$ However, ultrasound also has great potential for therapeutic applications at a higher power and is an emerging treatment applied in a range of clinical fields. ${ }^{2,3}$ Ultrasound can be widely used in ablative or nondestructive therapies, depending on the level of ultrasound energy provided. ${ }^{4}$ The number of possible therapeutic applications of ultrasound in neurological diseases continues to increase dramatically, as the potential of this method becomes more widely recognized.

Therapeutic ultrasound is now approved by the Food and Drug Administration (FDA) for use in the United States and in many countries outside of the United States for treatment of certain neurological diseases. However, its therapeutic applications are rapidly expanding for several neurological indications in humans. Not all clinical applications have reached the level of routine application in clinical practice. However, as transducer and computer control technologies have rapidly developed in recent years, the therapeutic 
applications of ultrasound are quite promising. Ultrasonic technology has shown very encouraging results for therapeutic application, and is at a highly advanced stage of research. In this review, we discuss the current clinical applications of therapeutic ultrasound, as well as the fields in which this promising technology is expected to be useful.

\section{BIOLOGICAL MECHANISM}

Ultrasound, under appropriate exposure conditions, can produce a wide range of biological effects in therapeutic application (Fig. 1). Individual ultrasound beams do not affect tissues as they pass through. However, the convergence of ultrasonic energy at a single point results in many important biological effects, depending on the tissue characteristics and ultrasound parameters. ${ }^{4}$ Focused ultrasound (FUS) uses concentrated ultrasound energy, where multiple beams intersect on a single point. Ultrasonic frequencies in the range of 20 $\mathrm{kHz}$ to about $3 \mathrm{MHz}$ are utilized in therapeutic ultrasound, whereas frequencies beyond these are used in diagnostic ultrasound. ${ }^{1}$

In therapy, ultrasound can induce effects not only through focal heating but also through non-thermal or mechanical effects, including cavitation, acoustic streaming, radiation forced tissue displacement, mechanical stress, or other undetermined non-thermal processes. ${ }^{2,3}$ Continuous ultrasound waves with high intensity can induce tissue ablation or hyperthermia, whereas non-thermal, pulsed ultrasound waves can induce neuromodulation, microbubble-mediated opening of the blood-brain barrier (BBB) to facilitate drug delivery to the brain, tissue healing, and immunomodulation. ${ }^{4}$ Several different mechanisms may contribute concurrently to the total biological effects of therapeutic ultrasound. ${ }^{2,3}$

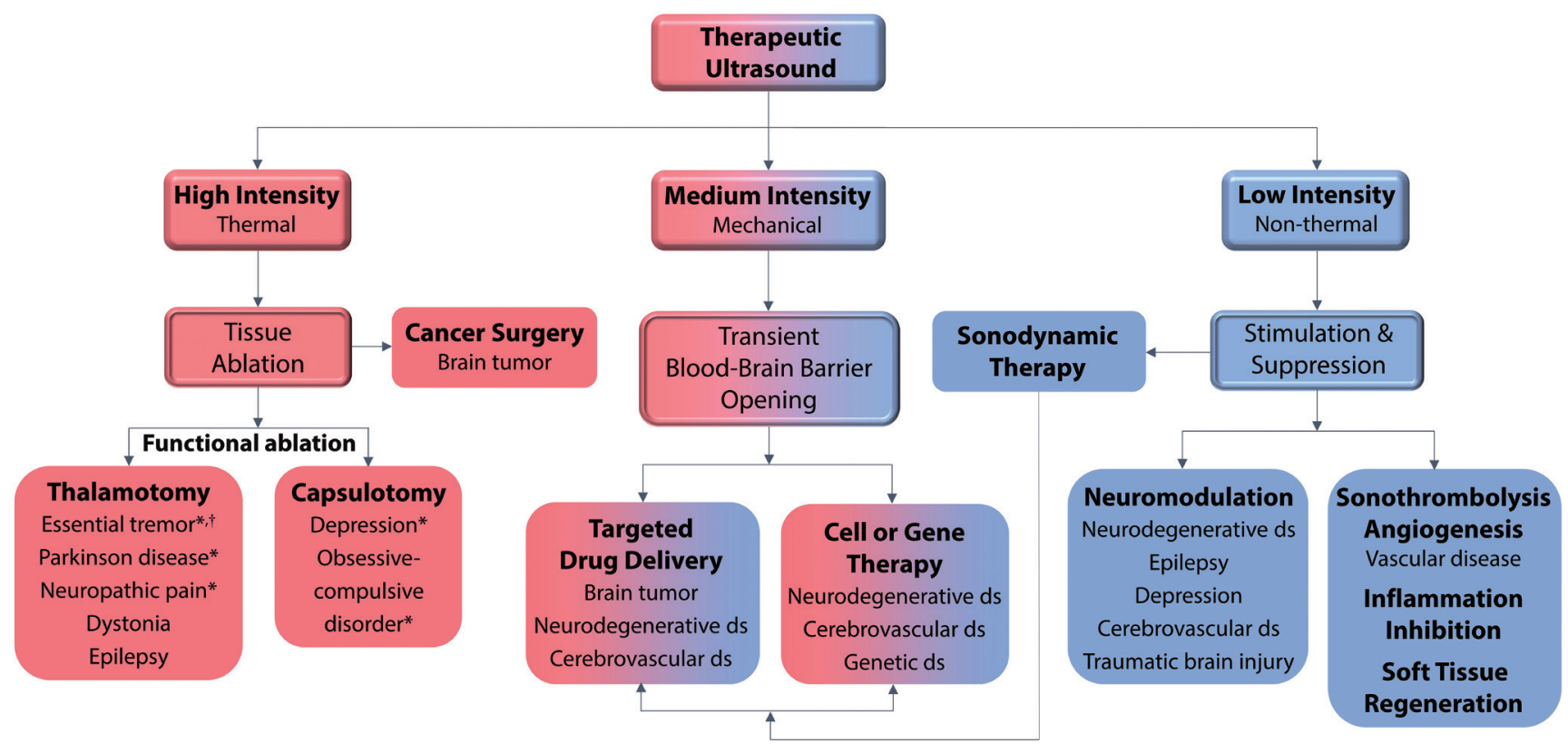

FIG. 1. Neurological diseases that can be treated in clinical practice, according to the biological mechanism of therapeutic ultrasound. Ultrasound can be widely used in ablative or nondestructive therapies, depending on the level of ultrasound energy provided. Therapeutic ultrasound has been approved in many countries, for the treatment of essential tremor, Parkinson's disease, neuropathic pain, depression, and obsessive-compulsive disorder $\left({ }^{\star}\right)$. The use of therapeutic ultrasound for the treatment of essential tremor has been approved by the United States Food and Drug Administration $\left({ }^{\dagger}\right)$. The number of possible therapeutic applications of ultrasound in neurological diseases continues to grow dramatically, as the potential of this method becomes more widely recognized. Therapeutic applications of ultrasound have the potential to significantly change treatment paradigms in neurological diseases. ds; disease. *Approved by countries outside the United States. ${ }^{\dagger}$ Approved by the United States Food and Drug Administration. 


\section{NEUROSURGERY}

\section{Brain tumor ablation and functional brain ablation}

Thermal ablation, which is the most direct mechanism of FUS, can be used to treat brain tumors. However, this has been limited to small case series because of concern that FUS, which is strong enough to induce thermal necrosis, carries an increased risk of intracranial hemorrhage (ICH), intracranial hypertension, and cerebral edema., 5 Furthermore, power limitations, and restriction of the target area by the surrounding sensitive brain tissue, prevent sufficient heating for tumor ablation. ${ }^{7,8}$ For these reasons, clinical trials using FUS for thermal ablation, focused on sites near the center of the brain, have not shown satisfactory results. Consequently, in contrast to tumor ablation, much research has been directed at neurosurgery using FUS for functional brain ablation, and there have been dramatic developments worldwide (Fig. 1).

Early exploration of FUS for clinical use was limited by beam distortion and energy absorption, because of the skull. However, advances in technology have allowed safety monitoring and confirmation of the energy being applied at the acoustic focus. ${ }^{2}$ More recently, FUS treatment has been monitored in real-time with magnetic resonance imaging (MRI) (MR-guided focused ultrasound [MRgFUS]). Thus, thermal lesioning can be performed accurately and evaluated immediately. ${ }^{9}$ This noninvasive procedure does not need scalp incision, bur hole or electrode penetrating to the brain, hence eliminating the risk of complications due to infection. Neurosurgery using FUS has made remarkable progress and is approved in the United States to treat essential tremor. Additional indications that have been approved outside of the United States are Parkinson's disease, neuropathic pain, depression and obsessive-compulsive disorder (Fig. 1).

\section{Essential tremor}

The most technologically advanced application of ultrasound, involving therapeutic functional brain ablation using FUS, is for the treatment of essential tremor. MRgFUS thalamotomy is an approved, effective, novel, and minimally invasive ablative treatment for essential tremor. In two pilot studies, essential tremor improved in patients treated with MRgFUS thalamotomy targeting the ventral intermediate nucleus (Vim) of the thalamus. ${ }^{10,11}$ A subsequent multicenter randomized control trial (RCT) in patients with medically refractory essential tremor showed that MRgFUS thalamotomy significantly improved tremor and disability scores compared to those of the placebo group. ${ }^{12}$ Adverse events in the MRgFUS treatment group included gait disturbance in $36 \%$, and sensory disturbances in $38 \%$, of patients.

Adverse events, and the effectiveness of MRgFUS for essential tremor treatment, could be highly dependent on the location and size of thalamotomy lesions. The area of optimal tremor response after MRgFUS thalamotomy was identified at the posterior portion of the Vim, whereas lesions extending beyond the posterior region of the Vim and lateral or inferolateral to the thalamus were associated with an increased risk for various acute adverse effects. ${ }^{13}$ Therefore, recent studies have explored the possibility of refining current MRgFUS treatment planning and targeting, thereby reducing side effects and improving clinical outcomes in patients with essential tremor, using various imaging technologies, such as diffusion tractography. ${ }^{14}$

\section{Parkinson's disease}

As the efficacy of MRgFUS for treatment of essential tremor has been proven, efforts to utilize MRgFUS for the treatment of non-essential tremor syndromes continue. Recent studies have demonstrated that MRgFUS in the Vim is a promising treatment to effectively treat tremors, other than essential tremor, in a wide range of disorders, especially Parkinson's disease. ${ }^{15-17}$ A recent RCT showed that FUS thalamotomy for patients with tremor-dominant Parkinson's disease improved medication-refractory tremor in these patients. ${ }^{17}$

Other studies have reported that pallidotomy using MRgFUS, i.e., lesioning of the globus pallidus interna or the fiber tracts exiting the pallidum en route to the thalamus (pallidothalamic tract), could also be an effective treatment for patients with Parkinson's disease. ${ }^{18,19}$ In treating Parkinson's disease is unclear as to which area is the best target, or whether different targets should be used for different patients, depending on disease symptoms. Future studies need to investigate 
these uncertainties and compare the outcomes of MRgFUS and deep brain stimulation, which is the current gold-standard surgical treatment option for medically refractory Parkinson's disease.

\section{Neuropathic pain}

Another clinical indication for ablative treatment in the thalamus using FUS is chronic medically refractory neuropathic pain. In fact, the first reported functional brain ablation application of MRgFUS was for the treatment of chronic neuropathic pain. Central lateral thalamotomy using MRgFUS in patients suffering from chronic medically refractory neuropathic pain resulted in satisfactory pain relief in $57 \%$ of patients after 1 year. ${ }^{20,21}$ However, there was one reported complication of bleeding in the study, and further clinical trials are underway to investigate the effectiveness and safety of MRgFUS thalamotomy for chronic neuropathic pain (NCTo1699477 and NCT03111277). The results of these studies need to be confirmed in the future.

\section{Neuropsychiatric conditions}

Bilateral capsulotomy using MRgFUS has shown significant improvement in the management of neuropsychiatric conditions, such as depression and anxiety. ${ }^{22,23}$ Previous studies have reported that functional brain ablation using MRgFUS targeting the anterior limb of the internal capsule improved neuropsychiatric symptoms in patients with medically refractory major depressive disorder and obsessive-compulsive disorder. ${ }^{22,23}$ Currently, several trials are underway that explore the use of MRgFUS in the treatment of medically refractory depression and obsessive-compulsive disorder (NCTo1986296, NCTo3156335, and NCTo2348411). The results of these trials are eagerly awaited and could lead to better control of these conditions.

\section{THERAPEUTIC DELIVERY}

\section{Transient blood-brain barrier opening}

Although not currently used in clinical practice, FUS-enhanced therapeutic delivery is an unlimited field for future exploration. Endothelial cells in the brain have continuous tight junctions that form the $\mathrm{BBB}$. This barrier is a major roadblock for the treatment of neurological disorders, due to the restricted access of many types of therapeutics from the bloodstream into the brain (Fig. 2). ${ }^{1,24}$ Therefore, therapeutic delivery across the BBB remains challenging. Non-thermal ultrasound, using mechanical mechanisms of action, can be used to manipulate opening of the BBB for the targeted delivery of drugs, genes, cells, antibodies, growth factors, or other agents to the desired area of the brain. ${ }^{25,26}$ By changing the sonication parameters to the pulsed mode, rather than the continuous mode used for ablation, localized, controlled, transient, reversible, and reproducible opening of the BBB can be achieved using ultrasound. ${ }^{26,27}$ This transient BBB opening can allow the systemic delivery of therapeutic agents, injected to locally sonicated areas of the brain, avoiding damage to surrounding neural tissue (Fig. 2).

FUS-induced BBB opening occurs via a process of cavitation, and injection of preformed microbubbles is performed to ensure biological effects, even at lower-energy sonication, and to reduce adverse effects on the surrounding neural tissue. ${ }^{25,28}$ The expansion of microbubbles, in response to ultrasound, displaces the vessel wall and transiently opens the tight junctions of the $\mathrm{BBB}$, thereby allowing drugs to enter the brain (Fig. 2). ${ }^{1,3,29}$ Previous studies have demonstrated the use of FUS to enhance the delivery of a number of drugs across the BBB in animals..$^{30-32}$ Additional studies have applied this technology to deliver other therapeutic agents, including stem cells, nucleic acids, and antibodies, for the treatment of neurodegenerative diseases in animals. ${ }^{33,34}$ Finally, results of a landmark trial using FUS to open the BBB in Alzheimer's disease patients temporarily showed that FUS was feasible and safe and could be used to open the BBB repeatedly. ${ }^{35}$ This trial is the first small, but critically important, step in a process that could potentially lead to a novel approach in treating Alzheimer's disease, by delivering therapeutic agents directly to the desired site of the brain. Further research via a phase 2 a trial, with a larger group of Alzheimer's disease patients, will be needed to determine the safety and efficacy of using FUS to breach the BBB.

Transient BBB opening, using therapeutic ultrasound, could be performed in almost all neurological 


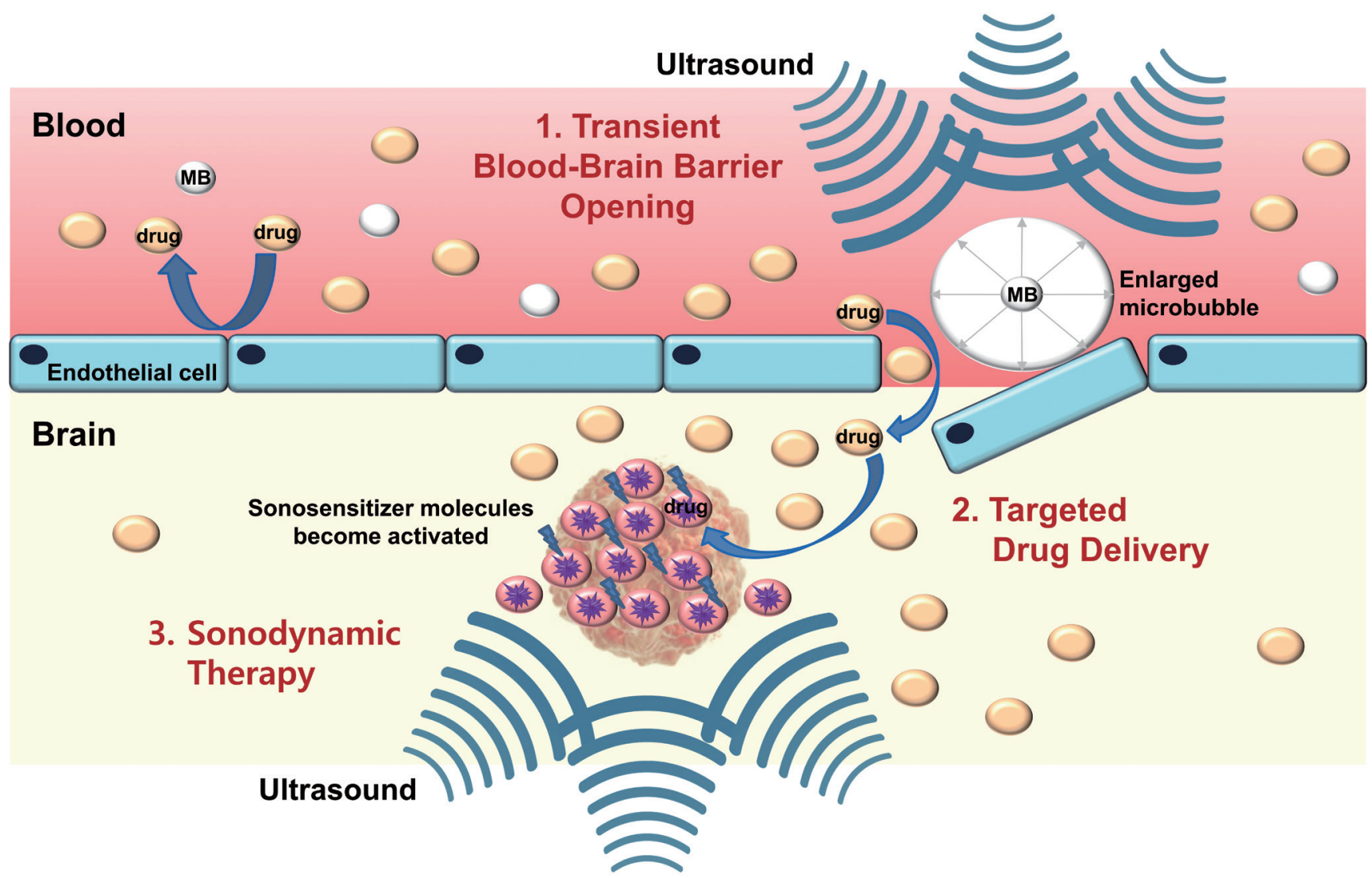

FIG. 2. Tight junctions of the BBB are a major obstacle to the effective delivery of potential therapeutic agents to the brain. When combined with microbubbles and ultrasound, expansion of microbubbles in response to ultrasound displaces the vessel wall and can temporarily open the BBB in a safe, localized, controlled, reversible and reproducible manner. Ultrasound-enhanced therapeutic delivery allows the passage of multiple drugs, neuroprotective agents, antibodies, circulating cells or even genes, to desired brain regions. Low-intensity ultrasound can activate therapeutic agents that have characteristics of sono-sensitizers, thereby enhancing the effect of treatment via sonoporation and sono-sensitization, known as sonodynamic therapy. BBB; blood-brain barrier, MB; microbubble.

diseases. Recently, another clinical trial showed that the novel technology of BBB opening using MRgFUS is feasible and safe in patients with primary brain tumors. ${ }^{36}$ If therapeutic agents can be safely delivered to the desired brain region with transient BBB opening using therapeutic ultrasound, options for many novel treatments will become available. These include amyloid-beta antibodies for treating Alzheimer's disease; genes or small interfering RNAs for treating genetic diseases; and stem cell therapy for treating ischemic, traumatic, and neurodegenerative diseases (Fig. 1).

\section{Sonodynamic therapy}

Low-power ultrasound has another beneficial effect in FUS-enhanced therapeutic delivery, as well as the temporary opening of BBB. Low-intensity ultrasound temporarily increases cell membrane permeability during exposure to ultrasound, known as sonoporation, and activates therapeutic agents via sono-sensitization (Fig. 2). ${ }^{37,38}$ Some therapeutic agents have characteristics of sono-sensitizers and exhibit synergistic interactions with ultrasound..$^{8}$ Treatment involving sonoporation and sono-sensitization using ultrasound is termed sonodynamic therapy (Fig. 2).$^{38,39}$ Currently, sonodynamic therapy is mainly studied and utilized in the field of cancer treatment. This therapy relies on sono-sensitizing compounds and FUS to generate intracellular reactive oxygen species, causing cancer cell death in brain tumors. ${ }^{40}$ In the future, however, therapeutic effectiveness in a wide variety neurological diseases could be enhanced by sonodynamic therapy (Fig. 1).37,39,40 


\section{NEUROMODULATION}

Low-intensity pulsed ultrasound can be used as a therapeutic tool for functional neuromodulation and alteration of neural activity, either activation or inhibition, similar to conventional neuromodulatory treatment. ${ }^{41}$ The currently used conventional neuromodulatory treatments have various disadvantages, such as their impact on the metabolism of pharmacological treatments and surgical procedures of electrode implantation for electrical treatments or deep brain stimulation. ${ }^{42}$ Conversely, previous studies have demonstrated reversible and non-invasive neuromodulatory and neuroprotective effects of low-intensity ultrasound on targeted brain regions, without elevating the temperature causing ablation..$^{41,42}$ Neuromodulation can also be achieved by the delivery of neuroactive substances to the desired area with transient BBB opening, using FUS (Fig. 2).

Therefore, neuromodulation using ultrasound is a promising potential application in the treatment of various neurological diseases (Fig. 1). Ultrasound could be effective for modulation of brain activity, including hippocampus and cortex activation, mood improvement, and inhibition of sensory paresthesia, depending on the targeted brain site. ${ }^{43,44}$ Recent studies have demonstrated that low-intensity ultrasound could be a powerful tool to prevent neuronal degeneration and can safely modulate the activity of cortical, thalamic and hippocampal circuits in humans as well as animals. ${ }^{44,45}$ These neuromodulatory effects might provide a novel therapeutic application of ultrasound to noninvasively target neurodegenerative and cerebrovascular lesions, or seizure foci (Fig. 1). Ultrasound-mediated neuromodulation could also be used for the treatment of neuropsychiatric conditions. Levels of brain-derived neurotrophic factor (BDNF) are reduced in depression, and this could be a fundamental cause of the condition. ${ }^{46}$ Evidence suggests that pulsed ultrasound stimulates neuronal circuits and increases BDNF levels in the hippocampus. ${ }^{42}$

The technological development of ultrasound will allow multiple brain regions to be targeted. These areas include deep-brain regions, like the amygdala for emotions, thalamus for attention, and brain stem for arousal and sleep. ${ }^{47}$ Superficial brain circuits, including the prefrontal cortex for decision-making, premotor cortex for movement planning, and posterior parietal cortex for spatial processing, could also be targeted. ${ }^{47}$ Although the effects on other brain areas and the underlying molecular and cellular changes are poorly understood at present, ultrasound-mediated neuromodulation could open new avenues for further enhancing the treatment of various neurological diseases.

\section{SONOTHROMBOLYSIS AND ANGIOGENESIS}

Ultrasound-induced thrombolysis and angiogenesis have shown potential for the treatment of vascular diseases, mostly in improving recanalization after vessel occlusion. ${ }^{48,49}$ In patients with acute ischemic stroke (AIS), high-frequency ultrasound significantly enhanced tissue plasminogen activator (tPA)-induced arterial recanalization, compared with tPA alone, and was found not to be associated with an increased risk of symptomatic ICH in a comprehensive meta-analyses and phase 2 trial..$^{4,50,51}$ Conversely, low-frequency and high-intensity ultrasound was associated with a high risk of hemorrhage..$^{2}$ These findings led to the conclusion that high-frequency ultrasound could be safe and effective for clot lysis in cases of AIS. ${ }^{1,48,49}$ However, recent preclinical studies have demonstrated that high-intensity FUS, rather than unfocused low-intensity ultrasound used in previous clinical trials, is a favorable option for thrombolysis with or without tPA.53 These results warrant further investigation in a clinical setting, by prospective RCTs.

In previous meta-analyses, high-frequency ultrasound-enhanced thrombolysis was associated not only with a higher likelihood of complete recanalization but also functional independence (defined as a 3-month modified Rankin Scale score of o-1 or o-2) when compared with tPA alone..$^{50,51}$ After the promising results of the previously mentioned phase 2 trial and meta-analyses, a phase 3 clinical RCT that was initiated to confirm the effect of sonothrombolysis on functional outcome was terminated, and the results of the trial have recently been released. ${ }^{54,55}$ Sonothrombolysis was reported to be feasible and most likely safe in patients treated with tPA after AIS. However, no clinical benefit was seen at 3 months after treatment. .55 
Clinical trials with microspheres for enhancing the effect of sonothrombolysis have also been conducted. In a recent RCT, sonothrombolysis with microspheres showed a trend toward higher recanalization and functional independence rates, compared with tPA alone. However, the treatment effect did not reach statistical significance. ${ }^{56}$ Other RCTs and meta-analyses have reported that sonothrombolysis with microspheres is safe and effective, leading to more complete recanalization and a trend toward better clinical outcomes. ${ }^{50,51,57,58}$ These results warrant further investigation into the role of sonothrombolysis with microspheres in the treatment of AIS with large vessel occlusion (LVO).

In patients with LVO-related AIS, the efficacy of endovascular treatment (EVT) is excellent, and it is therefore recommended as the standard treatment in current guidelines. ${ }^{59}$ However, sonothrombolysis could be applied in patients with LVO who are not candidates for EVT, in cases in which EVT has failed, or in countries where EVT is not yet offered as a standard of care. ${ }^{55}$ Sonothrombolysis may also enable clot lysis in intracranial vessel locations that are hard to reach, or present a high risk for perforation in EVT. Recently, a phase 3 clinical trial was initiated to evaluate the efficacy and safety of sonothrombolysis as an adjunctive therapy to tPA, before EVT, in patients with LVO (NCT03519737). The results of this study suggest that sonothrombolysis treatment, combined with EVT, could increase therapeutic effects.

Therapeutic ultrasound could also be useful for clot hemolysis in patients with spontaneous intracerebral hemorrhage..$^{53}$ In preclinical studies, MRgFUS has been reported to be feasible, fast, and efficient, as well as to allow safe sonolysis of $\mathrm{ICH} .{ }^{60}$ In addition, therapeutic ultrasound could increase angiogenesis following ischemic or hemorrhagic stroke, possibly even before intracranial angioplasty procedures. ${ }^{61,62}$ Thrombolysis and angiogenesis using ultrasound may have great capacity to complement current treatments in ischemic and hemorrhagic stroke (Fig. 1). However, further research is required to develop approaches that achieve the intended functional outcomes of sonothrombolysis and angiogenesis, without serious adverse events.

\section{INFLAMMATION INHIBITION AND SOFT TIS- SUE REGENERATION}

Low-intensity ultrasound could be a potential modality for inhibition of inflammation and promotion of soft-tissue healing (Fig. 1). ${ }^{63}$ Previous experiments have suggested that low-intensity ultrasound enhances the resorption of herniated disc and upregulates growth factor gene expression. ${ }^{64,65}$ Recent clinical studies have indicated that low-intensity ultrasound could encourage the recovery of cervical radiculopathy in patients. ${ }^{66,67}$ Pulsed ultrasound significantly improved thresholds of pain, after treatment in an animal model of chronic migraine. These results provide initial evidence that ultrasound may also provide an important therapeutic option for patients suffering from migraine. ${ }^{68}$ This novel treatment modality has attracted much attention because of the widespread applications and effectiveness of low-intensity ultrasound. ${ }^{63}$

\section{CONCLUSIONS}

Ultrasonography is widely recognized as a tool for imaging. However, recent research on therapeutic ultrasound has made great progress. In some neurological diseases, ultrasound has been used for therapeutic purposes in clinical practice, with proven efficacy. The use of therapeutic ultrasound for the treatment of essential tremor has been approved by the United States FDA. Therapeutic ultrasound has also been approved in many countries, for the treatment of Parkinson's disease, neuropathic pain and neuropsychiatric conditions, such as major depression and obsessive-compulsive disorder.

This modality has potential for use in the treatment of many more neurological diseases than currently being treated in clinical practice in the future. Therapeutic ultrasound has great potential for functional ablation of specific brain lesions, tumor ablation, neuromodulation, immunomodulation, sonothrombolysis, angiogenesis, and soft tissue healing. In addition, the effects of transient BBB opening in delivery of therapeutic agents and the subsequent use of sonodynamic therapy could revolutionize future treatment of neurological diseases. Considering the unlimited potential 
for the development of therapeutic ultrasound, clinical application could significantly change treatment paradigms in neurological diseases. However, further studies are necessary to ascertain the risks, advantages, and limitations of this emerging therapeutic modality.

\section{Acknowledgments}

This work was supported by a National Research Foundation of Korea grant, funded by the Korean Government (NRF-2019M3A9E8020261, K. H. C.).

\section{Conflicts of Interest}

No potential conflicts of interest relevant to this article was reported.

\section{REFERENCES}

1. Leinenga G, Langton C, Nisbet R, Gotz J. Ultrasound treatment of neurological diseases--current and emerging applications. Nat Rev Neurol. 2016;12:161-174.

2. Miller DL, Smith NB, Bailey MR, Czarnota GJ, Hynynen $\mathrm{K}$, Makin IR, et al. Overview of therapeutic ultrasound applications and safety considerations. J Ultrasound Med. 2012;31:623-634.

3. Goertz DE. An overview of the influence of therapeutic ultrasound exposures on the vasculature: high intensity ultrasound and microbubble-mediated bioeffects. Int J Hyperthermia. 2015;31:134-144.

4. Fishman PS, Frenkel V. Focused ultrasound: an emerging therapeutic modality for neurologic disease. Neurotherapeutics. 2017;14:393-404.

5. Medel R, Monteith SJ, Elias WJ, Eames M, Snell J, Sheehan JP, et al. Magnetic resonance-guided focused ultrasound surgery: part 2: a review of current and future applications. Neurosurgery. 2012;71:755-763.

6. Ram Z, Cohen ZR, Harnof S, Tal S, Faibel M, Nass D, et al. Magnetic resonance imaging-guided, high-intensity focused ultrasound for brain tumor therapy. Neurosurgery. 2006;59:949-955; discussion 955-956.

7. McDannold N, Clement GT, Black P, Jolesz F, Hynynen K. Transcranial magnetic resonance imaging- guided focused ultrasound surgery of brain tumors: initial findings in 3 patients. Neurosurgery. 2010;66:323-332; discussion 332.

8. Guthkelch AN, Carter LP, Cassady JR, Hynynen KH, Iacono RP, Johnson PC, et al. Treatment of malignant brain tu- mors with focused ultrasound hyperthermia and radiation: results of a phase I trial. J Neurooncol. 1991;10:271-284.

9. Ghanouni P, Pauly KB, Elias WJ, Henderson J, Sheehan J, Monteith S, et al. Transcranial MRI-guided focused ultrasound: a review of the technologic and neurologic applications. AJR Am J Roentgenol. 2015;205:150-159.

10. Elias WJ, Huss D, Voss T, Loomba J, Khaled M, Zadicario E, et al. A pilot study of focused ultrasound thalamotomy for essential tremor. N Engl J Med. 2013;369:640-648.

11. Lipsman N, Schwartz ML, Huang Y, Lee L, Sankar T, Chapman M, et al. MR-guided focused ultrasound thalamotomy for essential tremor: a proof-of-concept study. Lancet Neurol. 2013;12:462-468.

12. Elias WJ, Lipsman N, Ondo WG, Ghanouni P, Kim YG, Lee $\mathrm{W}$, et al. A randomized trial of focused ultrasound thalamotomy for essential tremor. N Engl J Med. 2016;375:730-739.

13. Boutet A, Ranjan M, Zhong J, Germann J, Xu D, Schwartz $\mathrm{ML}$, et al. Focused ultrasound thalamotomy location determines clinical benefits in patients with essential tremor. Brain. 2018;141:3405-3414.

14. Tian Q, Wintermark M, Jeffrey Elias W, Ghanouni P, Halpern $\mathrm{CH}$, Henderson JM, et al. Diffusion MRI tractography for improved transcranial MRI-guided focused ultrasound thalamotomy targeting for essential tremor. Neuroimage Clin. 2018;19:572-580.

15. Fasano A, Llinas M, Munhoz RP, Hlasny E, Kucharczyk W, Lozano AM. MRI-guided focused ultrasound thalamotomy in non-ET tremor syndromes. Neurology. 2017;89:771-775.

16. Zaaroor M, Sinai A, Goldsher D, Eran A, Nassar M, Schlesinger I. Magnetic resonance-guided focused ultrasound thalamotomy for tremor: a report of 30 parkinson's disease and essential tremor cases. J Neurosurg. 2018;128:202-210.

17. Bond AE, Shah BB, Huss DS, Dallapiazza RF, Warren A, Harrison MB, et al. Safety and efficacy of focused ultrasound thalamotomy for patients with medication-refractory, tremor-dominant parkinson disease: a randomized clinical trial. JAMA Neurol. 2017;74:1412-1418.

18. Na YC, Chang WS, Jung HH, Kweon EJ, Chang JW. Unilateral magnetic resonance-guided focused ultrasound pallidotomy for Parkinson disease. Neurology. 2015;85:549-551.

19. Magara A, Buhler R, Moser D, Kowalski M, Pourtehrani P, Jeanmonod D. First experience with MR-guided focused ultrasound in the treatment of Parkinson's disease. $J$ Ther Ultrasound. 2014;2:11.

20. Jeanmonod D, Werner B, Morel A, Michels L, Zadicario 
E, Schiff G, et al. Transcranial magnetic resonance imaging-guided focused ultrasound: noninvasive central lateral thalamotomy for chronic neuropathic pain. Neurosurg Focus. 2012;32:E1.

21. Martin E, Jeanmonod D, Morel A, Zadicario E, Werner B. High-intensity focused ultrasound for noninvasive functional neurosurgery. Ann Neurol. 2009;66:858-861.

22. Hurwitz TA, Honey CR, Allen J, Gosselin C, Hewko R, Martzke J, et al. Bilateral anterior capsulotomy for intractable depression. J Neuropsychiatry Clin Neurosci. 2012;24:176182.

23. Jung HH, Kim SJ, Roh D, Chang JG, Chang WS, Kweon EJ, et al. Bilateral thermal capsulotomy with MR-guided focused ultrasound for patients with treatment-refractory obsessive-compulsive disorder: a proof-of-concept study. Mol Psychiatry. 2015;20:1205-1211.

24. Pardridge WM. Drug transport across the blood-brain barrier. J Cereb Blood Flow Metab. 2012;32:1959-1972.

25. Sirsi S, Borden M. Microbubble compositions, properties and biomedical applications. Bubble Sci Eng Technol. 2009;1:3-17.

26. McDannold N, Vykhodtseva N, Raymond S, Jolesz FA, Hynynen K. MRI-guided targeted blood-brain barrier disruption with focused ultrasound: histological findings in rabbits. Ultrasound Med Biol. 2005;31:1527-1537.

27. Hancock HA, Smith LH, Cuesta J, Durrani AK, Angstadt $\mathrm{M}$, Palmeri ML, et al. Investigations into pulsed high-intensity focused ultrasound-enhanced delivery: preliminary evidence for a novel mechanism. Ultrasound Med Biol. 2009;35:1722-1736.

28. Hynynen K, McDannold N, Vykhodtseva N, Jolesz FA. Noninvasive MR imaging-guided focal opening of the bloodbrain barrier in rabbits. Radiology. 2001;220:640-646.

29. Sheikov N, McDannold N, Vykhodtseva N, Jolesz F, Hynynen $\mathrm{K}$. Cellular mechanisms of the blood-brain barrier opening induced by ultrasound in presence of microbubbles. Ultrasound Med Biol. 2004;30:979-989.

30. Liu HL, Hua MY, Chen PY, Chu PC, Pan CH, Yang HW, et al. Blood-brain barrier disruption with focused ultrasound enhances delivery of chemotherapeutic drugs for glioblastoma treatment. Radiology. 2010;255:415-425.

31. McDannold N, Arvanitis CD, Vykhodtseva N, Livingstone MS. Temporary disruption of the blood-brain barrier by use of ultrasound and microbubbles: safety and efficacy evaluation in rhesus macaques. Cancer Res. 2012;72:36523663 .
32. Sun T, Zhang Y, Power C, Alexander PM, Sutton JT, Aryal M, et al. Closed-loop control of targeted ultrasound drug delivery across the blood-brain/tumor barriers in a rat glioma model. Proc Natl Acad Sci U S A. 2017;114:E10281-E10290.

33. Burgess A, Ayala-Grosso CA, Ganguly M, Jordão JF, Aubert I, Hynynen K. Targeted delivery of neural stem cells to the brain using MRI-guided focused ultrasound to disrupt the blood-brain barrier. PLoS One. 2011;6:e27877.

34. Burgess A, Huang Y, Querbes W, Sah DW, Hynynen K. Focused ultrasound for targeted delivery of siRNA and efficient knockdown of Htt expression. J Control Release. 2012;163:125-129.

35. Lipsman N, Meng Y, Bethune AJ, Huang Y, Lam B, Masellis $\mathrm{M}$, et al. Blood-brain barrier opening in Alzheimer's disease using MR-guided focused ultrasound. Nat Commun. 2018;9:2336.

36. Mainprize T, Lipsman N, Huang Y, Meng Y, Bethune A, Ironside $\mathrm{S}$, et al. Blood-brain barrier opening in primary brain tumors with non-invasive MR-guided focused ultrasound: a clinical safety and feasibility study. Sci Rep. 2019;9:321.

37. Pan H, Zhou Y, Sieling F, Shi J, Cui J, Deng C. Sonoporation of cells for drug and gene delivery. Conf Proc IEEE Eng Med Biol Soc. 2004;5:3531-3534.

38. Kuroki M, Hachimine K, Abe H, Shibaguchi H, Kuroki M, Maekawa S, et al. Sonodynamic therapy of cancer using novel sonosensitizers. Anticancer Res. 2007;27:3673-3677.

39. Rosenthal I, Sostaric JZ, Riesz P. Sonodynamic therapy--a review of the synergistic effects of drugs and ultrasound. Ultrason Sonochem. 2004;11:349-363.

40. Prada F, Kalani MYS, Yagmurlu K, Norat P, Del Bene M, DiMeco F, et al. Applications of focused ultrasound in cerebrovascular diseases and brain tumors. Neurotherapeutics. 2019;16:67-87.

41. Tyler WJ. Noninvasive neuromodulation with ultrasound? A continuum mechanics hypothesis. Neuroscientist. 2011;17:25-36.

42. Tufail Y, Matyushov A, Baldwin N, Tauchmann ML, Georges J, Yoshihiro A, et al. Transcranial pulsed ultrasound stimulates intact brain circuits. Neuron. 2010;66:681-694.

43. Hameroff S, Trakas M, Duffield C, Annabi E, Gerace MB, Boyle P, et al. Transcranial ultrasound (TUS) effects on mental states: a pilot study. Brain Stimul. 2013;6:409-415.

44. Legon W, Sato TF, Opitz A, Mueller J, Barbour A, Williams A, et al. Transcranial focused ultrasound modulates the activity of primary somatosensory cortex in humans. Nat 
Neurosci. 2014;17:322-329.

45. Zhao L, Feng Y, Shi A, Zhang L, Guo S, Wan M. Neuroprotective effect of low-intensity pulsed ultrasound against MPP+-induced neurotoxicity in PC12 cells: Involvement of $\mathrm{K}_{2} \mathrm{P}$ channels and stretch-activated ion channels. Ultrasound Med Biol. 2017;43:1986-1999.

46. Duman RS, Li N. A neurotrophic hypothesis of depression: role of synaptogenesis in the actions of NMDA receptor antagonists. Philos Trans R Soc Lond B Biol Sci. 2012;367:24752484.

47. Tyler WJ, Lani SW, Hwang GM. Ultrasonic modulation of neural circuit activity. Curr Opin Neurobiol. 2018;50:222-231.

48. Alexandrov AV, Molina CA, Grotta JC, Garami Z, Ford SR, Alvarez-Sabin J, et al. Ultrasound-enhanced systemic thrombolysis for acute ischemic stroke. $N$ Engl J Med. 2004;351:2170-2178.

49. Daffertshofer M, Fatar M. Therapeutic ultrasound in ischemic stroke treatment: experimental evidence. Eur J Ultrasound. 2002;16:121-130.

50. Tsivgoulis G, Eggers J, Ribo M, Perren F, Saqqur M, Rubiera $M$, et al. Safety and efficacy of ultrasound-enhanced thrombolysis: a comprehensive review and meta-analysis of randomized and nonrandomized studies. Stroke. 2010;41:280287.

51. Saqqur M, Tsivgoulis G, Nicoli F, Skoloudik D, Sharma VK, Larrue $\mathrm{V}$, et al. The role of sonolysis and sonothrombolysis in acute ischemic stroke: a systematic review and meta-analysis of randomized controlled trials and case-control studies. J Neuroimaging. 2014;24:209-220.

52. Daffertshofer M, Gass A, Ringleb P, Sitzer M, Sliwka U, Els T, et al. Transcranial low-frequency ultrasound-mediated thrombolysis in brain ischemia: increased risk of hemorrhage with combined ultrasound and tissue plasminogen activator: results of a phase ii clinical trial. Stroke. 2005;36:1441-1446.

53. Zafar A, Quadri SA, Farooqui M, Ortega-Gutiérrez S, Hariri OR, Zulfiqar M, et al. MRI-guided high-intensity focused ultrasound as an emerging therapy for stroke: a review. $J$ Neuroimaging. 2019;29:5-13.

54. Schellinger PD, Alexandrov AV, Barreto AD, Demchuk AM, Tsivgoulis G, Kohrmann M, et al. Combined lysis of thrombus with ultrasound and systemic tissue plasminogen activator for emergent revascularization in acute ischemic stroke (CLOTBUST-ER): design and methodology of a multinational phase 3 trial. Int J Stroke. 2015;10:1141-1148.

55. Alexandrov AV, Köhrmann M, Soinne L, Tsivgoulis G, Bar- reto $\mathrm{AD}$, Demchuk AM, et al. Safety and efficacy of sonothrombolysis for acute ischaemic stroke: a multicentre, double-blind, phase 3, randomised controlled trial. Lancet Neurol. 2019;18:338-347.

56. Molina CA, Barreto AD, Tsivgoulis G, Sierzenski P, Malkoff MD, Rubiera M, et al. Transcranial ultrasound in clinical sonothrombolysis (TUCSON) trial. Ann Neurol. 2009;66:2838.

57. Molina CA, Ribo M, Rubiera M, Montaner J, Santamarina E, Delgado-Mederos R, et al. Microbubble administration accelerates clot lysis during continuous 2-MHz ultrasound monitoring in stroke patients treated with intravenous tissue plasminogen activator. Stroke. 2006;37:425-429.

58. Alexandrov AV, Mikulik R, Ribo M, Sharma VK, Lao AY, Tsivgoulis G, et al. A pilot randomized clinical safety study of sonothrombolysis augmentation with ultrasound-activated perflutren-lipid microspheres for acute ischemic stroke. Stroke. 2008;39:1464-1469.

59. Powers WJ, Rabinstein AA, Ackerson T, Adeoye OM, Bambakidis NC, Becker K, et al. 2018 guidelines for the early management of patients with acute ischemic stroke: a guideline for healthcare professionals from the American Heart Association/American Stroke Association. Stroke. 2018;49:e46-e110.

6o. Ilyas A, Chen CJ, Ding D, Romeo A, Buell TJ, Wang TR, et al. Magnetic resonance-guided, high-intensity focused ultrasound sonolysis: potential applications for stroke. Neurosurg Focus. 2018;44:E12.

61. Mu HM, Wang LY. Effect of therapeutic ultrasound on brain angiogenesis following intracerebral hemorrhage in rats. Microvasc Res. 2015;102:11-18.

62. Lu ZY, Li RL, Zhou HS, Huang JJ, Qi J, Su ZX, et al. Rescue of hypertension-related impairment of angiogenesis by therapeutic ultrasound. Am J Transl Res. 2016;8:3087-3096.

63. Jiang X, Savchenko O, Li Y, Qi S, Yang T, Zhang W, et al. A review of low-intensity pulsed ultrasound for therapeutic applications. IEEE Trans Biomed Eng. 2018 Dec 25 [Epub]. https://doi.org/10.1109/TBME.2018.2889669.

64. Kobayashi Y, Sakai D, Iwashina T, Iwabuchi S, Mochida J. Low-intensity pulsed ultrasound stimulates cell proliferation, proteoglycan synthesis and expression of growth factor-related genes in human nucleus pulposus cell line. Eur Cell Mater. 2009;17:15-22.

65. Iwabuchi S, Ito M, Hata J, Chikanishi T, Azuma Y, Haro H. In vitro evaluation of low-intensity pulsed ultrasound in herniated disc resorption. Biomaterials. 2005;26:7104-7114. 
66. Sayilir S. The short-term effects of TENS plus therapeutic ultrasound combinations in chronic neck pain. Complement Ther Clin Pract. 2018;31:278-281.

67. Metin Ökmen B, Ökmen K, Altan L. Investigation of the effectiveness of therapeutic ultrasound with high-resolution ultrasonographic cross-sectional area measurement of cervical nerve roots in patients with chronic cervical ra- diculopathy: a prospective, controlled, single-blind study. $J$ Med Ultrason (2001). 2018;45:479-486.

68. Walling I, Panse D, Gee L, Maietta T, Kaszuba B, Kumar V, et al. The use of focused ultrasound for the treatment of cutaneous allodynia associated with chronic migraine. Brain Res. 2018;1699:135-141. 